Revue internationale P.M.E.

Économie et gestion de la petite et moyenne entreprise

Revure

internationale

PME

\title{
Innovation et communication sociale dans les districts industriels
}

\section{Maria Tinacci Mossello et Francesco Dini}

Volume 2, numéro 2-3, 1989

URI : https://id.erudit.org/iderudit/1007937ar

DOI : https://doi.org/10.7202/1007937ar

Aller au sommaire du numéro

Éditeur(s)

Presses de l’Université du Québec

ISSN

0776-5436 (imprimé)

1918-9699 (numérique)

Découvrir la revue

Citer cet article

Tinacci Mossello, M. \& Dini, F. (1989). Innovation et communication sociale dans les districts industriels. Revue internationale P.M.E., 2(2-3), 229-251.

https://doi.org/10.7202/1007937ar
Résumé de l'article

L’analyse de l'articulation régionale des procès généraux de développement a mis en évidence comment de nouveaux facteurs «immatériaux» de localisation (les relations, la culture) vont remplacer les facteurs traditionnels physiques en offrant de nouveaux éléments aux processus localisés circulaires et fondamentalement stables basés sur l'importance historique de faits socio-économiques. Le cas des districts industriels italiens revalorise les dynamiques persistantes d'autoréférence. Soutenus par une forte propension à l'innovation et par la qualité territoriale d'un système de valeurs parallèle interagissant (comme l'expliquent les économies externes marshalliennes d'agglomération) ces districts représentent un modèle de développement sur lequel il paraît opportun de réfléchir, en regard des éléments critiques qui émergent de l'analyse de cas concrets. 


\title{
Innovation et communication sociale* dans les districts industriels
}

\author{
Maria TINACCI MOSSELLO \\ et Francesco DINI** \\ Université de Florence
}

\begin{abstract}
RÉSUMÉ
L'analyse de l'articulation régionale des procès généraux de développement a mis en évidence comment de nouveaux facteurs aimmatériaux" de localisation (les relations, la culture) vont remplacer les facteurs traditionnels physiques en offrant de nouveaux éléments aux processus localisés circulaires et fondamentalement stables basés sur l'importance historique de faits socio-économiques. Le cas des districts industriels italiens revalorise les dynamiques persistantes d'autoréférence. Soutenus par une forte propension à l'innovation et par la qualité territoriale d'un système de valeurs parallèle interagissant (comme l'expliquent les économies externes marshalliennes d'agglomération) ces districts représentent un modèle de développement sur lequel il paraît opportun de réfléchir, en regard des éléments critiques qui émergent de l'analyse de cas concrets.
\end{abstract}

- Recherche partiellement financée par le Conseil National de recherche italien no 85.01050.08. Même si cette recherche provient d'une réflexion commune, les parties 1 et 2 ont été préparées plus particulièrement par Maria Tinacci Mossello alors que les parties 3,4,5,6,7 l'ont été par Francesco Dini.

* Maria TINACCI MOSSELO est professeur titulaire de Géographie économique à l'Université de Florence; elle a effectué des recherches dans plusieurs champs en s'orientant au cours des années récentes, vers les études sur la régionalisation et sur les rapports entre l'économie de la production et celle de l'environnement. Francesco DINI est licencié en Sciences politiques à la Faculté Cesare Alfieri de Florence et travaille dans le secteur des marchés de l'industrie privée et en particulier sur le fonctionnement des systèmes territoriaux des petites et moyennes entreprises en Italie. Adresse: Dipartimento di Scienze Economiche, Sezione di Geografia Economica - Via Curtatone, 1 - 50100 Firenze. 


\begin{abstract}
The analysis of the regional subdivision of the general development processes has emphasized how some new «immatérialı localization factors (relations, culture) are replacing the traditional psysical factors by giving new nourishment to circular localized processes and tendentially stable based on the historized substance of socioeconomic facts. The case of the Italian industrial districts enlightens immense and persistent self-reference dynamics. Supported by a strong propensity to innovation and by territorializing quality of a parallel system of values interacting to the classical Marshall's economies of agglomeration, they represent a development model on which it seems advisable to reflect, also in connection with critical elements emerging from the analysis of concrete cases.
\end{abstract}

\title{
RESUMEN
}

El analisis de la articulacion regional de los procesos generales de desarrollo ha puesto en evidencia como los nuevos factores «Inmateriales" de localizacion (las relaciones, la cultura) van sustituyendo los factores tradicionales materiales, dando nuevo alimento a processos localizados circulares y con tendencia de estabilidad basados sobre el peso historico de factores socio-economicos. El caso de los distritos italianos industriales pone en luz dinamicas autoreferenciales ingentes y persistentes, apoyados por una fuerte propension a la innovacion e interactante en las clasicas economicas marshallianas de aglomeracion, ellas constituyen un modelo de desarrollo sobre el cual es interesante reflexionar, tambien en relacion a los elementos criticos que surgen del analisis de casos concretos. 


\section{Introduction}

La nouvelle phase d'industrialisation technique, caractérisée par le développement et la réduction des coûts de transport et par la transformation du processus du travail (devenu moins accablant et moins localisé, tout en restant toujours soumis aux lois économiques) pose en particulier un double problème à propos des processus généraux du développement régional et de leurs articulations. En premier lieu, on sait que les facteurs matériels de localisation (l'abondance de l'eau et des ressources minérales, la productivité agricole, l'accessibilité géographique) deviennent de moins en moins importants par rapport à ceux immatériels reliés essentiellement aux communications et à la culture; ces facteurs représentent des composantes sociospaciales clés, procurent sur le territoire des «avantages» influençant le cours du développement et créent des processus circulaires localisés et à tendance stable et qui produisent de la synergie entre les processus généraux de développement et le territoire.

Cette situation, du point de vue de la recherche, nous oblige à rendre nos analyses dynamiques pour comprendre ces processus et à considérer la régionalisation soit comme une composante, soit comme une résultante de ces processus. L'indéniable complexité des caractéristiques du développement du territoire dérive de la coexistence de plusieurs processus qui divergent selon leur orientation téléologique, leur vitesse, la persistance de leurs caractères historiques et ainsi de suite.

La recherche d'une solution épistémologiquement intermédiaire entre l'approche générale et celle régionale a déjà été discuté chez les géographes italiens il y a quelques années (Pecaro, 1960; Ortolani, 1963). Puis, des apports théoriques plus pertinents sont venus de disciplines autres que la géographie, en particulier de l'économie politique et de la théorie des systèmes appliquée à la recherche sociale. La première a fourni, avec les travaux d'Alfred Marshall, l'important concept du district industriel; la seconde a donné celui du «système» social et de là, du «système socioterritorial». L'un et l'autre de ces modèles ont été repris de façon efficace par les géographes pour analyses des contextes généraux régionaux fortement dynamiques.

L'oeuvre de Marshall reprise par Becattini et son école (Becattini, 1987) ouvre des horizons fort intéressants pour étudier les liaisons entre le développement économique et le territoire, à partir du concept des économies externes. Ainsi, le district industriel se caractérise, du côté de la production, par la présence d'un grand nombre de petits producteurs suffisamment proches et réciproquement reliés à l'intérieur d'un processus de production subdivisé en de nombreuses phases. Du côté de l'environnement de la production, l'analyse marshallienne parvient à intégrer la culture industrielle (commune au district) et à dépasser l'hypothèse restrictive du district homogène et spécialisé, en mettant à nouveau en évidence les économies externes produites par les différentes firmes, par la complémentarité sur le marché du travail et par la demande de consommation qui en découle (Marshall, 1966; Bellandi, 1987). Chose importante, la structure socio-économique située au centre de cette 
analyse marshallienne dépasse l'aspect production pour intégrer la famille et à travers celle-ci, toute la communauté territoriale. Cette communauté devient ainsi produit et productrice du territoire; elle s'explique par le territoire et y induit des comportements homogènes donnant son sens de l'appartenance et fournissant toute la coopération nécessaire.

D'un autre côté, la théorie des systèmes fournit à l'analyse des structures industrielles un langage extraordinairement efficace pour l'étude des synchronismes (les relations) et des désynchronismes (les traditions, la finalité) de chaque structure. Cette idée holiste n'est pas étrangère à la tradition de la géographie (Vallega, 1982); mais elle trouve particulièrement un fondement logique dans l'existence de structures territoriales «fortes» et tendanciellement persistantes, permettant de comprendre les processus de transformation et de développement. Cette greffe de la théorie systémique en géographie (Vallega, 1976 et 1982; Turco, 1984 et 1988) permettant de fascinants isomorphismes provient de l'oeuvre de Bertalanffy (1968) et des travaux de Luhmann (1983a) en référence aux systèmes sociaux ${ }^{1}$. Les concepts d'équifinalité, d'ouverture, de complexité, d'auto-référence, d'autoréaction, ont permis de développer une vision plus avancée du problème soulevé.

\section{La construction historico-sociale du district industriel}

Le district industriel s'est construit en un temps qui ne s'exprime pas simplement comme mécanique, mais comme temps historique, ou mieux, comme un temps mesuré selon les règles dialectiques du développement, de l'organisation et de l'entropie. C'est un temps qui a besoin de temps. Ce jeu de mots est là pour souligner qu'il n'existe pas un mètre chronologique pour mesurer la profondeur historique d'un district, ni pour préciser le nombre d'années pour qu' une industrie établie dans un lieu déterminée finisse par devenir typique et unique. Pour Marshall, les premiers mobiles de cette installation industrielle relèvent des caractéristiques géographiques, histo-

1. «La théorie des systèmes sociaux provenant de nombreuses contributions et représentant une extension des travaux de Parsons, est une théorie de type structurel-fonctionnel. Ceci signifie que le concept de structure précède celui des fonctions... Ce faisant, la théorie structurelle-fonctionnaliste se prive de toute possibilité de questionner les structures et d'affronter non seulement le problème du sens dans le développement des structures, mais encore celui du sens de la formation même des systèmes». Il importe donc de proposer une théorie fonctionnelle-structuraliste, qui inverse le rapport et permet de s'interroger sur «le problème du fonctionnement des structures systémiques sans devoir présupposer l'existence d'une structure systémique complexe comme point de référence au même problème» (Luhmann, 1983a, 130-131). Une telle proposition constitue une nouvelle approche systémique qui permet de dépasser la distinction entre structure et processus, à travers la procédure de réduction de la complexité. 
riques et politico-psychologiques de la région; mais lorsque ce choix est fait, il est probable que c'est pour longtemps, grâce aux économies externes dont l'industrie jouit avec le temps. Ces économies sont :

$1^{\circ}$ la diffusion des spécialités et du savoir faire;

$2^{\circ}$ le renouvellement des inventions et des innovations;

$3^{\circ}$ le développement du commerce et des transports;

$4^{\circ}$ les contacts étroits avec les fournisseurs et les acheteurs;

$5^{\circ}$ le développement de la complémentarité des différentes phases (du processus de production) et des différents spécialistes (produits);

$6^{\circ}$ l'élargissement du marché du travail spécialisé (soutenu par l'immigration);

$7^{\circ}$ le renouvellement de la capacité entrepreneuriale, etc.

Lorsque le développement régional se consolide de façon à créer des économies externes technologiques, l'aire industrielle concentrée devient vraiment système, ou un ensemble, selon Bertalanffy (1968), téléologiquement ordonné, de faible entropie, grâce au cercle vertueux de l'organisation/information/innovation ${ }^{2}$.

Au début, le temps n'a pas besoin seulement de temps, mais de continuité; et le vecteur de cette continuité apparaît moins radical dans ses composantes physiques que dans ses aspects socio-culturels du territoire. La première composante de l'organisation économico-sociale qui génère des stocks et des rendements croissants est le travail, facteur de production vivant. Celui-ci ne peut être réduit à une marchandise; il repose sur l'existence humaine et implique un environnement culturel relié non seulement au lieu de production mais à tout le territoire. Ceci produit le résultat du rapport nécessaire et interactif entre le travail et l'espace, dans le sens que le travail organise l'espace et est à la fois qualifié et orienté sur le territoire d'où il agit.

Evidemment, les analyses et la politique, fascinées par les règles simplistes de l'économie classique, ont à la longue sous-évalué et réduit à des catégories marginales ou déviantes les régions socio-spatiales. Quoique les formes du développement économique (uniquement) régional ne sont pas toujours uniformes, il est difficile de nier que ces analyses sont concordantes et conformes à l'hypothèse de la concurrence parfaite; elles relèvent, d'un côté, du modèle économico-politique de l'accumulation capitaliste et, de l'autre, de celui du développement économique polarisé. On recourt dans ces cas à des explications qui reviennent à la théorie néoclassique de la production et en particulier à la théorie du travail, dans laquelle le marché du travail n'est qu'un ensemble indifférencié, analogue à une quelconque

2. L'ouverture du système régional produit des effets négentropiques; en référence aux éléments constituant la structure territoriale, cela veut dire qu'une telle ouverture permet l'apport d'énergie et d'information, sous forme d'investissements, d'innovations et, surtout, d'immigrations. C'est ce que Marshall (1919) soulignait par la capacité particulière des zones de fort développement industriel spécialisé à attirer de l'énergie s'ajoutant à celle d'origine locale. 
marchandise et où le travail n'est qu'un facteur de production mobile et mû par son prix, sans égard à son contexte social. (Becattini, 1979b; Storper et Walker, 1983). Il est vrai que dans le cadre néo-classique, on a reconnu que «certaines» industries nécessitaient du travail spécialisé, en particulier en étudiant les zones d'industrialisation ancienne et les grandes villes (Hoover, 1948; Pred, 1978) et que les recherches italiennes ont souvent mis le territoire au centre des analyses (exemple : Bagnasco, 1977). Mais à l'encontre de la réalité, ces études ont considéré que de telles spécialisations étaient géographiquement stables ou n'ont pas tenu compte suffisamment du changement dans les tissus régionaux.

La régionalisation de la production et de son développement relève des analyses de l'organisation territoriale; elle trouve son fondement dans les conditions de fonctionnement du travail dans la production, qu'on peut résumer en utilisant les termes de disponibilité, contrôle et créativité. Ces caractéristiques tirées de situations particulières s'intègrent comme une série de conditions sociales développées dans l'environnement culturel auquel appartiennent les travailleurs - sécurité, santé, régularité de l'emploi, carrière, mobilité sociale et ainsi de suite - et sont confrontées dans la production selon un mécanisme de conflit/coopération.

Le travail en effet est un facteur productif conscient qui peut développer les attitudes antagonistes ou coopératives non seulement en rapport avec le type de relations industrielles en vigueur dans le secteur et le «lieu» de production, mais encore en relation avec les valeurs socio-culturelles développées dans l'appartenance à la communauté, en fonction de la qualité du travail ou selon le niveau de vie général dans lequel les travailleurs s'insèrent (Walker et Storper, 1981).

Face à l'offre de travail, de tout ceci, on peut dire qu'il se dévelopope un fort lien localisé avec la reproduction des facteurs de travail, qui ne se limite pas à l'entreprise mais s'étend à l' habitat et à la communauté; les travailleurs sont des produits de la société beaucoup plus que de l'industrie. Ces éléments géographiques irréductibles de la reproduction de la force de travail permettent d'expliquer dans différentes régions la stabilité du point de vue culture et éthique de travail et sont à l'origine des différences de capacité, d'autonomie et de créativité.

D'un autre côté, l'enracinement régional explique aussi l'évolution localisée au moins à deux niveaux. D'une part, il facilite l'ouverture régionale pour acquérir l'énergie et l'information : en intrants il y a l'immigration, l'attraction des investissements, l'offre d'innovation; en extrants, il y a les phénomènes de décentralisation, quoique des relations industrielles dans la communauté rendent plus probables les investissements internes au système.

D'autre part, le second niveau se réfere à la capacité sui-référencielle du système, dans le sens que ses dynamismes se développent selon un code de connexion propre au système et capable d'utiliser les informations provenant de l'extérieur sans perdre sa propre «vie», tout en se différeciant de l'environnement humain plus large (Luhmann, 1983a, 1983b). En particulier, dans le district industriel, la culture industrielle sui-référentielle trouve tant sa stabilité que son dynamisme dans le 
développement d'un apprentissage diffus, mais aussi extra-commercial, grâce à l'atmosphère dont parle Marshall et à un processus d'innovation endogène largement radical et fonctionnant par «learning by doing and by using» (Tinacci Mossello, 1987a).

\section{Le district industriel comme système sui- référenciel}

L'interaction entre le niveau économique et le niveau social constitue donc le trait saillant tant des districts industriels observésà la fin du dix-huitième siècle par Alfred Marshall que du district industriel italien comme il existait dans la seconde moitié du 20ème siècle. Bien qu'on ne puisse évidemment pas expliquer le district industriel avec cette seule caractéristique, il y a de bonnes raisons pour considérer qu'elle soittypique. De même que les économies externes marshalliennes constituent la clef pour expliquer le phénomène d'agglomération spatiale, de même le système particulier de valeurs distinguant chaque territoire est aussi la clef pour éclairer ce qu'on peut appeler les mailles serrées de l'organisation sociale du district industriel.

Ce système de valeurs opère à deux niveaux. Le premier niveau maintient la cohérence entre les fins individuelles, cohérence qui permet la réponse subjective à la finalité intersubjective qui se manifeste dans la communauté. A un deuxième niveau, ce mécanisme communautaire générant les normes comportementales et la régulation pour les garantir, finit par transmettre d'une génération à l'autre le corps de valeurs et par circonscrire les conflits d'intérêts des acteurs «à l'intérieur de l'intérêt communautaire intériorisé dans le complexe de population locale» (Becattini, 1989b; 5) ou du «système local de vie et de travail» (Bellandi, 1989b). Le district industriel se comprend donc de l'intérieur et de ses confrontations avec l'extérieur, ou comme une entité intimement unitaire; les diverses définitions, comme cercles virtueux, atmosphère marshallienne, ou système, ne font qu' interpréter simplement les différentes façons d'observer une réalité fortement unitaire.

D'un autre côté, aujourd' hui le district industriel peut se voir comme un tissu de relations spatiales aussi consistantes et qui remet en question l'interprétation du paradigme classique de centre/périphérie (Tinacci Mossello, 1987b; Dematteis, 1989). L'expérience italienne de cette localisation particulière de la petite industrie, à la différence de ce qui s'est passé dans d'autres zones de l'Europe méditérranéenne (Garofoli, 1989), s'est normalemènt caractérisée durant cette dernière décennie par un penchant déterminé pour l'innovation; et ceci a permis d'éviter les conséquences du déclin de tout cycle de production défini par la théorie.

Examinons maintenant le problème de l' autoréférence du district industriel (ceci en recouvrant spécifiquement au concept de la complexité dans les analyses du monde moderne et post-moderne) et des relations entre les catégories socio-territoriales des district industriels présents sous le thème (introduit durant les années $70 \mathrm{par}$ 
Luhmann) des procédures de réductions de la complexité environnementale comme dynamisme d'équilibre d'un système. Un système social est un système typiquement ouvert dans le sens de Bertalanffy (1968), et, comme tel, on peut penser qu'il est doté d'équifinalité. A la différence d'un système fermé qui tend à s'équilibrer à travers un parcours forcé, un système ouvert peut parvenir à un stade d'équilibre à travers plus d'opportunités ou de parcours alternatifs. Dans l'isomorphisme de Luhmann (1983a; 134-138), un système social est stable s'il y a plus à perdre de se maintenir, qu'à gagner à changer. Un système social — dans notre cas, un système socio-territorial - est d'autant plus complexe que seront nombreuses les solutions pour répondre aux sollicitations de l'environnement et grande la capacité de réaction.

Cette ambivalence est la condition de stabilité d'un système social, qui lui permet d'accroître sa propre complexité afin de contrôler sa stratégie potentielle et, en même temps, de réduire et simplifier cette complexité afin de dominer leséléments internes qui tendent à imposer leur propre stratégie.

En effet, dans le district industriel, cette réduction de la complexité environnementale - qui, selon Luhmann (1983a; 145-151) équivaut au devenir de la structure du système - peut être drastique. Ainsi, en observant le milieu industriel du district, il est facile de reconnaitre que la monoculture industrielle d'un seul modèle productif, fondé sur l'homogénéité de la base, réduit la complexité du système. Une si faible diversification productive et de composition limitée du cycle du travail dans l'unité locale, lorsqu'elle est strictement interdépendante, ne confere évidemment pas un grand degré de liberté. Au contraire, plus stricte est une telle interdépendance, plus grandes sont les économies d'agglomérations qui alimentent le système. De plus, la personnalisation des contacts dus à la contiguïté, qui constitue un des «mécanismes» les plus importants du district (Dei Ottati, 1987; Bellandi, $1989 \mathrm{~b}$ ), assure la circulation de l'information selon des modalités et un langage «spécialisé», et rend problématique (Zanfei, 1987) leur traduction selon d'autres codes de communication (exemple : le code télématique). Dans cet optique, le contenu de la complexité du district industriel est objectivement insuffisant pour être absorbé vu le grand nombre de relations qu'il développe avec son environnement. Non seulement le cercle virtueux du district produit un système au contenu insuffisant pour l'équifinalité, mais il requiert de la multistabilité (Luhmann, 1983a; Turco, 1988) encore insuffisamment observée. D'un autre côté, en termes d'isomorphisme entre le naturel, le social et le territorial, le système est sui-référenciel, c'est-à-dire qu'il est capable de se référer à ses propres mécanismes circulaires et de promouvoir l'organisation qui alimente ses tensions lui permettant de durer. «Le système» n'attend pas et ne s'attend pasà des intrants informationnels provenant de l'extérieur pour faire varier sa structure; mais il génère de façon autonome, grâce à sa perception et à sa mémoire, les mécanismes autorégulateurs nécessaires.

Toute société représente un système sui-référenciel qui tend donc à être :

«a) ouvert à l'environnement en termes cognitifs, du moment qu'il possède et développe sa capacité d'intégrer un flux d'informations (stimulations, perturba- 
tions) provenant de l'extérieur, b) fermé à l'environnement en termes normatifs, lorsqu'il contient en soi des mécanismes soutenant sa propre existence et est à un niveau pour se réaliser de façon autonome» (Turco, 1988 : 124-145).

Une telle cohérence de substance ressemble à celle propre au district industriel. Sa nature sui-référencielle nous permet en réalité de voir le district industriel par rapport à l'information extérieure ayant pour but de limiter sa complexité, d'un côté, comme partiellement ouvert de façon cognitive - indépendamment de sa capacité à complexifier sa propre monoculture industrielle ou son propre modèle productif et sa propre hiérarchie de valeur - et de l'autre, comme hermétiquement fermé de façon congnitive.

\section{$4 \quad$ La communication sociale dans le district industriel}

Le filtrage de l'information par les districts industriels ne signifie toutefois pas que celle-ci n'existe pas. Le district est naturellement exposé à toute l'information extérieure. La monoculture industrielle tendra cependant à en écarter une grande partie qui n'apparaît pas utile à sa finalité et à «traduire» le reste en un langage propre au processus sui-référenciel. A l'encontre de la pluralité typique de langage des systèmes complexes, le district industriel limite la traduction de toute l'information qui entre à ce qui est nécessaire à ses propres fins. Une telle façon de faire a déjà été observée dans les interactions entre le niveau économique et le niveau social d'un territoire spécifique. Il est en effet clair que la réduction de la complexité - soit la réduction des opportunités autres - et de la rigidité du code normatif ne peut assurer la stabilité qu'en présence d'éléments méta-économiques qui outrepassent le simple effet des économies d'agglomération. L'intensité de cette «fermeture» normative rappelle directement l'autre isomorphisme géographique du processus auto-créateur (Turco, 1988; 124-134). Directement adapté de la biologie (Maturana et Valera, 1980; Zeleny, 1981), un tel processus constitue la «sublimation» de «l'autoréférencialité» et permet au système de réaliser son auto-construction et son «individualisation» progressive par rapport à son environnement. Dans le cas du district industriel, dont l'origine provient au tout début d'un projet économique individuel ou peu partagé, ce processus (soit la réduction de la complexité de l'environnement) d'individualisation génère une dynamique qu'on peut dire centripète. Celle-ci se réalisera à l'intérieur du système d'autant plus rapidement que la sélection de l'information sera importante. Le district industriel possède donc une complexité «pauvre» en terme de pluralité des prestations offertes, mais une complexité forte et sui-référencielle lorsqu'il auto-produit une structure capable d'absorber les effets négatifs des perturbations et de les transformer en facteurs d'ordres. Cet ensemble d'éléments fonctionnels, qui représente les normes et les institutions du système, lui 
permettra de se structurer ou de se restructurer de façon organique et de consolider son processus. Et c'est dans cette logique que se réalise l'interaction typique entre le niveau économique et le niveau social à travers un processus (soit toujours la réduction de la complexité de l'environnement) qui implique la structuration des valeurs particulières au district.

La communauté du district industriel s'individualise par rapport à l'extérieur en générant des valeurs communes intersubjectives et étroitement liées à la monoculture industrielle; ces valeurs soutiennent les différents aspects de la vie communautaire en favorisant l'approfondissement même du processus.

Ainsi, la réduction de la complexité de l'environnement accompagne la réduction de la complexité des valeurs. Ceci limite les conflits d'intérêts et produit le consensus. Cette métaphysique des valeurs a des références plus concrètes par exemple au niveau de «l'éthique du travail et de l'activité» (Becattini, 1989b), fondée sur la mobilité sociale qui, selon les catégories sociologiques, peut être rapide et peut favoriser la destinée individuelle de personnes qui se connaissent bien; ou encore elle peut être, par la famille, le poumon qui permet de respirer au rythme de la conjoncture économique, garantissant ainsi la flexibilité au système; enfin, elle explique l'approbation ou la réprobation sociale qui permet d'accepter dans la communauté ceux qui adhèrent au modèle et de repousser les autres.

Un tel mécanisme de tri d'information externe peut modifier sensiblement les valeurs qui entrent. Mais ce n'est pas le cas de nombreux districts industriels italiens situés dans les régions du Centre-Nord-Est qui sont gouvernés historiquement par le parti Communiste et qui sont aussi insérés dans un développement d'autocréation mythique («métopoietico»).

L'information qui entre dans le district industriel pénètre à l'intérieur d'une logique qui recouvre tant l'information relative au processus économique (la production, la commercialisation) que celle relative au processus social ${ }^{3}$. Dans ce cas, les deux dynamismes sont strictement interdépendants, et toute information qui entremême indépendante de la dynamique productive et commerciale - est traduite en langage afférent aux fins de la production du district. En réalité, «tous les principaux aspects de la vie sont en quelque sorte affectés».

L'information génère et est produite dans des appareils de régulation : «les institutions comprennent naturellement le marché, l'entreprise, la famille, l'église et l'école, mais aussi l'administration publique, l'articulation locale des partis politiques, des syndicat et de plusieurs autres organisations publiques, d'assistances et culturelles, religieuses et artistiques. Afin que le métabolisme social puisse fonction-

3 La relation entre l'information relative au processus social (la communication sociale) et l'information relative au processus productif peut être considérée comme un cas typique de rétroaction, reliée à la réflexivité des processus des systèmes sociaux. 
ner sans problèmes graves, ces institutions du district doivent être plus ou moins toutes perméables à ce même système de valeurs» (Becattini, 1989b; 4).

Une telle configuration est un ensemble convergent et en même temps en contradiction avec la lecture du processus de territorialisation ${ }^{4}$ proposé par Raffestin (1984). Celui-ci avance que la substrat de la territorialisation réside dans les valeurs, de sorte que le territoire s'individualise en se fermant selon des jugements de valeurs. L'émergence du mode de production industrielle et post-industrielle partage cependant une territorialisation fixée par le caractère politique-religieux (une «regula» comprise dans le sens mythologique du terme et donc immuable et totalement indivisible) d'une territorialisation mouvante (par une «auto-régula» du marché qui consiste en un système de signes qualifiant la seule valeur d'échange). La territorialisation - manifestée par des frontières — n'est pas plus régie par la régula qui génère l'espace régionalisée que par la croissance d'un système de relations sociales progressivement instables et complexes. Ce processus naturel rend stratégique la variable temporelle par rapport à celle spatiale; et ainsi la nature de la territorialisation devient essentiellement dynamique, se «territorialisant et se réterritorialisant incessamment au niveau de la logique territoriale hétérogène».

Convergence et contradiction constituent essentiellement la curieuse transposition des termes dans lesquels nous avons défini le processus de l'autocréation mythique («metopoietico»). Bien que le district industriel en tant que tel, soit gouverné par les règles économico-classiques de l'offre et de la demande, cette adhésion aux éléments spécifiques des valeurs tend à générer une «régula» particulière, qui s'applique à l'intérieur d'un territoire spécifique propre à ces valeurs et qui entraîne la stabilité des économies d'agglomération. Aussi, si la «régula» fixe la territorialisation, dans un continuum de déterritorialisation/reterritorialisation, le district industriel tend une fois de plus à se caractériser par une forte intégration sociale et par un fort encouragement à la production (entendu comme fournissant les conditions concrètes de production, à l'encontre d'une économie complexe abstraite). Cette «régula» qui détermine de façon précise les limites territoriales du district serait néanmoins une forme pure de «l'auto-régula» du marché, qui tend à «envahir» toute la société.

4 Le processus de territorialisation «déterritorialisation» reterritorialisation (Raffestin, 1984), peut être relié de façon étroite au «principe de complexité à partir de la rumeur» de Atlan, pour lequel l'auto-organisation du système génère des réponses aux perturbations externes et aux fluctuations internes, et se constitue à travers des séquences de désorganisation-réorganisation que la globalité du système n'a pas investie. La stabilité du système dérive, en effet, des relations (instables) qui lient les changements (et les retours) aux niveaux hiérarchiques. 


\section{L'innovation dans le district industriel}

Le mécanisme de «régula» de fermeture normative du système pour garantir son équilibre - qui, naturellement, n'est pas seulement un équilibre interne, mais surtout un équilibre des confrontations par rapport à l'externe - peut s'ouvrir de façon cognitive selon un mécanisme d'homéostasie par rapport à l'environnement. Le district industriel doit conserver sa capacité autorégulative dans sa confrontation avec le flux d'information nécessaire pour soutenir son processus productif; sa structure monoproductive doit en effet démontrer sa capacité à absorber les perturbations provenant de l'extérieur, tout en même temps assurer sa cohérence interne. Une telle évolution, en effet, soutient directement, bien que parfois d'une façon peu visible, ses propres mécanismes qui déterminent la «régula».

C'est pourquoi on peut trouver un très grand nombre d'études sur l'innovation dans les districts industriels (Sabel, 1982; Russo, 1985; Tinacci Mossello, 1987b; Bellandi, 1989a et 1989b); et cette innovation peut être liée aux concepts de «learning by doing» et de «learning by using» (Rosenberg, 1982). Cette «capacité d'innovation diffuse» (CID) est en effet un des éléments qualifiant le mieux l'autoréférentialité du système et constitue un phénomène complémentaire qui peut être substitué à l'activité de recherche et développement typique des organisations plus complexes (par exemple celle de la grande entreprise en tant que catégorie économique, ou de la grande ville en tant que catégorie spaciale). Ce sont en effet des caractéristiques spécifiques du district industriel, soit la perception globale du processus productif interne de la part des agents, la consolidation des liens entre le processus productif et celui commercial, l'habitude de conditions de travail instables et la capacité à prévoir rapidement les opportunités et les stimulants (Bellandi, 1989a et 1989b).

Dans le district industriel, le plus souvent les innovations ne requièrent pas des langages et des procédures aussi complexes que dans les grandes entreprises concurrentes, puisque la base productive et les processus sont homogènes; de plus, elles ont une plus grande probabilité de résoudre un grand nombre de problèmes concrets et communs à plus d'une firme.

Elles s'appliquent plus rapidement et entraînent des résultats mesurables presqu'immédiatement. Les avantages permettent de compenser le retard temporel ou de complexité comparée aux grandes entreprises.

Cette CID typique du district est l'indice d'une ouverture cognitive qualitativement importante. Elle se réalise en grande mesure par la saisie et l'adaptation - dans le cas des innovations, de produits et de processus - les informations qui entrent, en particulier celles provenant des modifications exogènes de la demande ou des opportunités offertes par de nouvelles technologies réalisées plus ou moins dans les secteurs de spécialisation du district.

Les analyses microéconomiques ont mis en évidence les potentialités et les limites de la CID. Bien que l'intensité de l'innovation évolue vraisemblablement à 
l'encontre du cycle économique (Giovannelli, 1982), la pratique continue de l'innovation permet quelquefois de garantir une évolution technologique graduelle : $c$ 'est le cas, par exemple, des districts industriels en Emilie-Romagne, qui ont ainsi réussi à surpasser la concurrence des nouveaux pays industriels (Corée du Sud, Taiwan...). Ici entre en jeu, un mécanisme non précisément économique, soit «l'orgueil d'être le premier» (Becattini, 1989b), et qui renvoie à l'interaction typique entre le niveau économique et le niveau social du district. Non seulement la hiérarchie des valeurs ou l'éthique de travail et de l'activité, mais la communication sociale réussit à créer des conditions adaptées pour faciliter cette capacité d'innovation diffuse.

Dans ce sens, la propension endogène à l'innovation constitue un des mécanismes centraux de l'autorégulation du système, qui se fonde sur l'élargissement des relations fonctionnelles entre ses éléments propres - «entendus comme agents économiques et comme agents sociaux». Dans un tel mode de régulation, les hypothèses du modèle de «filtering down» de la diffusion de l'innovation sont démenties; le district industriel manifeste ainsi une vivacité créative particulière, capable d'absorber et de fournir une réponse à ses propres carences structurelles en R \& D.

\section{$6 \quad$ Le cas de Prato}

\subsection{Le processus évolutif}

Situé à l'intérieur du réseau des formations sociales des milieux typiques de la «Terza Italia», le cas du textile pratésien peut être considéré comme un district industriel arrivé à «maturité». La monoculture industrielle s'est en effet diversifiée à l'intérieur, le cycle productif s'est désagrégé générant un phénomène intense d'entrepreneuriat diffus, l'atmosphère industrielle et l'interaction entre l'économie et le social s'est consolidée. Toutefois, la part de la monoculture du textile est en déclin par rapport au développement du tertiaire et des services aux entreprises.

Ainsi, le Prato constitue un large espace où on retrouve une entreprise par vingt habitants parmi les 14000 entreprises du textile existantes. Le développement de cette production de base a été très rapide. Le recensement 1981, par rapportà celui 1951 , a vu tripler les employés et multiplier par vingt le nombre d'entreprises locales. Après la guerre, s'est donc mis en place le dynamisme sui-référentiel du district et se sont développées de nombreuses situations critiques à laquelle la capacité autorégulative du système a dû apporter une réponse.

Du point de vue de la structure de production, on a observé la prolifération des entreprises locales et la diminution de leur taille moyenne - actuellement de quatre employés ou moins - par décomposition du processus productif. Le proces- 
sus, qui a favorisé radicalement ce nouvel aménagement productif local, a généré un maximum de flexibilité (Nigro, 1982; Giovannelli, 1984) et a anticipé de quelques années la décentralisation de la production qui a touché une grande partie de l'industrie italienne des années 70 . Cela a produit une mutation significative de l'état et de la logique du système.

Initialement, on a vu se constituer un noyau d'unités productives semiintégrées, alimentées par la demande d'un tissu d'entreprises artisanales locales et sous-traitantes, et favorisant la formation des premiers travailleurs industriels. Entre ces unités et les travailleurs, on a vu aussi arriver cette figure typique entrepreneuriale de «l'impannatore»" cet intermédiaire précurseur chargé de transmettre l'information et de garantir l'équilibre entre l'offre et la demande. Ensuite, la structure s'est orientée progressivement vers les petites entreprises, jusqu'à l'extrême fragmentation du cycle de production. Les grandes entreprises ont perdu progressivement leur capacité de contrôle sur le marché à l'avantage des «impannatore», alors que se multipliait le nombre d'entreprises locales artisanales sous-traitantes. La logique d'un tel changement repose sur la réduction progressive des productions de masse, sur l'avantage des petites séries, sur le renouvellement continu des échantillons auprès d'acheteurs potentiels pour s'assurer un positionnement stratégique sur les marchés, sur des produits toujours anticipés (par exemple, à base de design ou de qualité des fibres) et sur des relations commerciales particulières (le respect rigoureux des commandes, des possibilités de remises, etc.). Aussi ces changements dans la structure interne du district peuvent se justifier à l'externe, suite à l'évolution des conditions dans l'environnement. La demande, typiquement liée au facteur changeant de la mode, est passée du besoin de tissus de lainage cardé lourd, adapté à la production en séries et de masses, à des tissus progressivement plus légers, dessinés et moins dépendants de la laine.

En ce sens, les innovations les plus importantes du district ont porté avant tout sur le produit. Déjà, dans les années 50 , on assiste à une première diversification entre la filature «cardée» et celle «peignée», à l'introduction des fils de fantaisie pour les tricots; à la fin des années 70, lorsque l'innovation se porte sur les tissus estivals, la laine devint marginale et les fibres utilisées sont de plus en plus variées.

Cette évolution est devenue tellement importante qu'elle transforme la production des entreprises locales au point de démentir l'image traditionnelle du Prato, capitale de la guenille et des tissus usagés que les entreprises recyclent et employent pour leur production. Un tel changement atteint son maximum dans les

5 «L'impannatore» constitue bien l'image historique du district pratésien. «Sa fonction spécifique est de traduire en termes des produits vendables tout le potentiel offert à travers le tissu historique du district. Le district industriel est pour «l'impannatore» une sorte de capital malléable, capable de produire toutes sortes de biens dans un domaine spécifique. Sa principale immobilisation consiste dans sa connaissance particulière d'un certain district et, éventuellement, ses annexes et ses dépendances» (Becattini, 1989b : 11). 
années 70 et permet sans doute de développer des avantages compétitifs permettant au district de s'emparer de la quote part de marché détenue auparavant par l'Allemagne et la Grande-Bretagne (Pollard, 1982). Actuellement, $2 \%$ seulement des entreprises locales oeuvrent dans le recyclage et les «guenilles», ce qui ne fait pas plus de $10 \%$ de la matière première utilisée dans le district (Balestri et Toccafondi, 1988; Balestri 1989).

On ne peut donc parler d'une conjoncture dans laquelle l'information provenant de l'extérieur (la mode, les modalités des relations industrielles) est utilisée pour permettre d'adapter la structure de production à une dynamique qui accepte de remettre en question les produits, entreprises, processus et configurations sociales. Le nouveau stade atteint favorise une nouvelle situation dans laquelle le Prato, en changeant de type d'entreprises locales, consolide un processus d'entrepreneuriat diffus et crée un état de niveau supérieur dans un contexte d'évolution progressive globale de l'histoire de la «matrice laniera», Les tissus estivals, en effet, demandent des fibres telles que le lin, le coton, la soie, la viscose, etc alors que les tissus de lainage, à la fin des années 60 , représentaient encore $90 \%$ de la production locale (Balestri, 1989).

Mais cette nouvelle situation est, évidemment, en déséquilibre par rapport au supposé modèle théorique qui prévoit la décomposition du cycle productif chez les entreprises locales du district et semble anticiper la crise que le district vivra à partir de la moitié des années 80, alors que les faillites d'entreprises seront plus importantes que les créations et que l'emploi diminuera pour la première fois. Cette orientation vers les tissus légers, en effet, a entraîné l'ouverture du système vers l'extérieur, parce que les nouvelles fibres ne viennent plus de la production locale. Ceci a rompu l'intégration du système productif qui favorisait les entreprises artisanales et sous-traitantes; et cette situation s'est révélée problématique en créant des stimulis à l'innovation technologique que soutenait le mécanisme d'autorégulation du système de ces entreprises artisanales et sous-traitantes. Ce processus d'innovation - soutenu par le système bancaire local - favorise la redondance progressive de l'offre face à une demande en baisse, avec comme conséquence des faillites et la chute de la demande de main d'oeuvre (Tinacci Mossello, 1987 : 6).

Le district est donc en train de vivre une phase de consolidation des entreprises industrielles qui tendent à un contrôle majeur du marché et réduisent la complexité du processus de production du district en contrôlant mieux chacune des phases et la demande extérieure. A ceci, s'accompagne la restructuration de la base productive des entreprises artisanales et sous-traitantes, en difficulté avec la crise du lainage. Il semble cependant que la réponse à la conjoncture des années 70 - où la recherche d'une plus grande flexibilité et d'un meilleur processus d'entrepreneuriat diffus - n'entraîne actuellement aucune des rigidités du modèle productif. En particulier, la technologie de produits et de processus reliée à l'utilisation de la microélectronique (CAO et FAO) s'appliquent difficilement à un cycle de production décomposée entre trop d'entreprises qui requièrent de nombreuses modalités en 
terme de logistiques et des opérations de gestion complexes. Ceci naturellement ne signifie pas que le district pratésien ne peut plus innover, mais que l'innovation même devient particulièrement difficile. Cette difficulté structurelle survient avec la réorientation de la demande de la part des grandes entreprises donneur-d'ordre, entraînant un processus en chaîne défavorable.

La conjoncture actuelle peut se résumer ainsi : la stratégie de décentralisation de la production et du contrôle a été fonctionnelle face à l'information qui entre (la mode, les modalités des relations industrielles) et aux informations qui sortent ('’offre d'une grande flexibilité, des produits incorporant innovation et service). Mais ceci apparaît actuellement en contradiction avec l'état actuel des informations qui entrent (l'évolution de la mode et des styles d'habillement, le potentiel technologique difficilement applicable, la nouvelle concurrence de la part des nouveaux pays industriels) et des informations qui sortent

De façon hypothétique, on peut dire que la décentralisation productive était nécessaire entre les années 50 et 70 pour soutenir l'interaction entre le niveau économique et celui social; mais aujourd'hui on assiste à une recherche des mécanismes d'autorégulation qui contredisent une telle hiérarchie des valeurs (Lorenzoni, 1979). C'est-à-dire qu'on assiste à la rupture du cercle vertueux et à un phénomène de ségrégation progressif ${ }^{6}$ où une partie du système s'est complexifié et s'appuie vers l'extérieur alors que l'autre tend à se redimentionner. Mais une telle hypothèse, règle ordinairement le futur avec un langage du passé. Rien n'assure, en effet, que la crise des entreprises artisanales et sous-traitantes n'a pas «libéré» des ressources réutilisables par le système en mode sui-référentiel, à l'exemple du secteur tertiaire qui croit au Prato plus rapidement que la moyenne nationale (Tinacci Mossello, 1987). En fait, le système est maintenant majoritairement ouvert vers l'extérieur et le modèle productif dans la conjoncture actuelle prévoit une forte réduction des économies d'agglomérations. Une telle hypothèse peut être mesurée en référence à l'expérience particulière du projet SPRINT.

\subsection{Le projet SPRINT}

Le projet SPRINT (Système d'innovation technologique de Prato) lancé en 1983 et en opération depuis, constitue une des expériences européennes d'une intervention intégrée dans un territoire aux caractéristiques semblables à celles du

6 Le phénomène de ségrégation progressive a été mise en évidence par Bertalanffy et a été formalisée dans la fonction «alométrique» de nature exponentielle. Dans une vision ontologique du système, cette ségrégation progressive implique une rupture dans la dynamique auto-régulative et la perte de la qualité systémique. En réalité, selon la cohérente de la dynamique des systèmes naturels (Atlan, 1979, 1985; Prigogine et Nicolis, 1977) le mécanisme auto-régulateur d'un système socio-spatial peut fonctionner en forçant ou en solutionnant les conflits d'intérêts entre les divers éléments-agents du système (Vallega, 1982, 121-131). 
district industriel. Initié par «l'Unione Industriale Pratese», qui regroupe les entreprises guides des deux associations d'artisants ${ }^{7}$ et qui représentent à la base la soustraitance, et par l'Enea (Comité Nationale pour la recherche et le développement de l'énergie nucléaire et des énergies alternatives), le projet a reçu successivement l'adhésion de tous les autres agents politiques et économiques opérant sur le territoire (la municipalité, la fédération locale des Syndicats du Textile, les banques locales et la Chambre de Commerce de Florence, soit de la province dans laquelle est situé Prato), auxquels se sont jointes la STET ${ }^{8}$ et les autres firmes de crédit actives dans le district. La région de Toscane même si elle ne fait pas partie de l'Association qui gère SPRINT assure le financement de $20 \%$ du budget, alors que $47 \%$ est à la charge de l'Enea et le reste, $33 \%$, est garanti par les autres associés.

Cette société un peu disparate prouve son importance dans l'ampleur de ses objectifs. SPRINT s'est articulé en effet dans trois projets : un «projet télématique», un «projet infra-technologique» et un «projet énergétique». Après six ans, le résultat est difficile à juger sur la base des expériences en cours souffrant de plusieurs contradictions. Le «projet énergétique» ayant pour but d'épargner l'énergie et d'améliorer les conditions de l'environnement n'a jamais vraiment décollé et se trouve actuellement bloqué par un trop faible budget qui limite les choix. Le «projet infra-technologique» qui devait favoriser l'innovation au travers des activités de formation et de recherche a dû être en pratique modifié; après une phase d'espérimentation limitée sur un programme de CAO et d'une commande électronique pour un métier circulaire en bonneterie, il a été réorienté, de l'étude d'une technologie de production (teinture et filature), à celle d'une technologie sur l'organisation et la gestion; il semble que ces études permettront en 1991 d'appliquer de nouvelles technologies de contrôle-qualité et d'automatisation logistique des processus productifs. Le «projet télématique», démarré avec l'ambition de «globaliser» la «ville/ entreprise» Prato, se trouve actuellement à gérer un ensemble de services pour un petit groupe d'usagers, qui ne touche pas plus de $2 \%$ des organismes locaux.

Par contre, il faut dire que ces études ont donné des résultats non négligeables d'une certaine accumulation locale de savoir-faire, même s'il est difficile de nier la faillite vis-à-vis des objectifs de départ. Il est donc intéressant d'analyser les causes des difficultés rencontrées jusqu'à présent et ainsi d'émettre quelques hypothèses d'explication sur le Prato en particulier et sur le district industriel en général.

On peut trouver un double niveau de contradictions dans le fonctionnement du projet SPRINT. Le premier niveau concerne les relations entre le Prato entendu comme système et l'extérieur, le second touche aux relations internes au système même.

7 La C.N.A. (la Confédération Nationale des Artisans) et la Confartigianato (la Confédération des Artisans Italiens) constituent les deux associations nationales d'artisans. La première est orientée politiquement à gauche, la seconde au centre.

8 La Stet (la Société Finanzira Telefonico per Ozioni) est la société financière publique qui contrôle la SIP, détentrice du monopole national des télécommunications. 
A l'époque où les trois associations, acceptaient la proposition de l'Enea, le système de Prato se montrait cognitivement ouvert aux confrontations avec l'extérieur et favorisait particulièrement l'initiative. Le système devait cependant inévitablement confier le projet à des ressources externes. On a donc eu recours à la «Software-house», mais surtout on a dû accorder le rôle de maitre-d'oeuvre aux organismes Enea et Stet. Pour ces organismes, aux intérêts plus larges, le projet SPRINT ne figurait pas comme une fin, mais comme un moyen ou comme un laboratoire permettant l'application future de l'expérience à d'autres régions. On peut faire l'hypothèse, par conséquent, que ces objectifs ne permettaient pas dès le départ de maintenir le délicat équilibre du district pratésien, mais se référait au modèle abstrait de n'importe quel district industriel. Prenons le cas du choix du modèle Vidéotex de la part de la Stet qui a entraîné deux conséquences négatives. En premier lieu, la gestion de l'information n'est pas locale, et le flux d'informations provient et retourne à Milan. En second lieu, le Vidéotel (le modèle Vidéotex de la Stet) n'est pas compatible avec aucune autre entreprise pratésienne déjà dotée du programme E.D.P. Les décisions qui ont été prises selon les règles - ayant comme norme de favoriser l'intérêt général - et qui confiaient à l'administration publique le monopole des télécommunications, ont eu cependant des conséquences multiples : le système de Prato n'a pas pu faire valoir de façon suffisamment forte ses propres objectifs sui-référentiels dans un projet qui pourtant le concernait. On trouve ici, évidemment, un problème de taille d'évaluation politique qui a fait en sorte que le district industriel n'a pu faire prévaloir ses intérêts spécifiques. Ce refus, à l'intérieur de la dynamique même du district, est lié à une mauvaise anticipation de la part des agents du district, pour gérer adéquatement un tel conflit d'intérêt. L'ouverture cognitive d'un système n'entraîne pas nécessairement une réponse de fermeture auto-régulative et permet à nouveau de voir deux niveaux générant deux processus distincts d'auto-référencialité; un premier processus lié à la fraction culturellement plus avancée de la base productive et qui tend à favoriser le progrès et un deuxième processus relié à l'unité locale commerciale et sous-traitante, qui se manifeste en termes de fermeture cognitive et qui tend à perpétuer les modèles traditionnels de communication.

Le second niveau de contradiction concerne les relations internes dans ce même système. En fait, le «projet télématique» fournit avec ses objectifs la meilleure réponse possible à la reconfiguration conceptuelle et neutre du système. En assurant l'inter-connexion totale entre l'unité locale dans le territoire, il détermine en effet la complète transparence de l'information. Le «modèle du marché» favorise le partage total des dynamismes et de la créativité et génère, dans les faits, un modèle démocratique d'auto-contrôle. Les sous-traitants dénonceront, après quelque temps, les nouvelles conditions pour produire qui les obligent à informer les firmes industrielles sur ce qu'ils font et comment ils le font, et les compagnies de transport sur leur disponibilités quotidiennes et sur leurs déplacements. On en arrive ainsià une situation sui-référentielle qui débouche sur l'ubiquité de l'information et sur les meilleures opportunités pour l'alimenter.

Au moment de la réalisation, toutefois, les deux organismes se sont heurtées 
à deux intérêts opposés, liées à la double articulation du processus productif du système. D'un côté, les industriels les plus importants ont eu peur de perdre le contrôle ou leur pouvoir provenant des limites dans la transparence de l'information; ils ont donc refusé de soutenir la formalisation d'un modèle qui prévoyait mesurer à priori leur propre demande et ainsi d'expliquer la source de l'efficience complexe du système gérée par leur fonction de leader, dans leur confrontation avec l'évolution de la demande internationale. De l'autre, les sous-traitants, à leur tour, ont refusé de «modéliser» une telle relation de dépendance en considérant que cette façon de révéler l'information leur serait préjudiciable. Le «projet télématique» a été ainsi vidé de son contenu de départ et s'est transformé graduellement en services indépendants auprès des organismes intéressés. Actuellement, il y a le service «Uniform» de l'Union industrielle pratésienne, qui fournit des données sur le niveau de confiance de la clientèle (basées sur une enquête auprès de 100 entreprises sur les 2000 existantes), divers modèles de gestion administrative des services de traitements informatiques pour la fiscalité et le crédit, et d'autres services de consultation aux deux associations d'artisans et des services pour chacune d'entre elles (environ 150 firmes affiliées sur les 14000 existantes). A ceci, on a successivement joint un service d'information de la municipalité sur les services publics disponibles, un service de débit direct par les firmes locales de crédit (environ 300 entreprises affiliées) et enfin un service d'information sur des travailleurs locaux du syndicat du textile, leur disponibilité à temps plein ou à temps partiel et leurs possibilités de déplacement. Un autre service, ouvert à qui le veut, est le service Pratel, c'est-à-dire une banque de données, encore très incomplète, et qui fournit des informations générales sur les capacités techniques et productives d'un nombre limité d'entreprises. Actuellement, la stratégie des agents locaux est d'essayer de ramener à Prato la gestion globale des flux d'information et de développer l'échange d'information systématique pour toucher, en deux ans, environ 800 entreprises (soit moins de $15 \%$ des firmes locales, mais une fraction très significative des entreprises-guides) et à développer des services pour une clientèle plus instruite, tel que, par exemple, les professionnels.

Cette expérience permet de confirmer le processus de type allométrique qu'a introduit le système externe, lorsque les changements dans la demande internationale sont entrés en contradiction, à partir des années 50 , avec la structure contingente du système industriel bâti dans les 30 années. Durant cette période, il semble que le fonctionnement du système obéissait à une sorte de macro-dynamisme autorégulé et organique aux divers sous-systèmes dans lesquels s'articulait le district pratésien. Les bouleversements successifs ont conduit à une individualisation progressive des sous-systèmes; alors que leur dynamique autorégulée résultait de la diversité culturelle souple ou de l'ouverture cognitive des agents étroitement liéesà l'évolution productive. Dans ce sens on peut trouver dans tout cela un des noeuds du problème en émergence dans le district pratésien : les entreprises «de pointe» - celles qui, par exemple, ont eu rapidement recours au télécopieur - se sont ouvertes à l'information et ont ainsi réorienté leur production vers l'extérieur; alors que pendant ce temps les artisans et les sous-traitants avaient un intérêt insuffisant dans l'innovation télématique. 


\section{Conclusion}

Le cas de Prato permet de mettre en lumière une série de phénomènes contrastés (mais non contradictoires) qui confirme dans ces systèmes industriels la persistance d'un fonctionnement significativement sui-référentiel en tant que logique spécifique permettant la lecture de faits socio-territoriaux et du potentiel de différenciation et de territorialisation. L'évolution de cette dynamique auto-organisative, activée par la nouvelle technologie électronique et télématique, permet, en effet, de stratifier le système et de redéfinir son contenu et ses limites. Mais les conséquences sur les réactions du milieu, en termes fonctionnels, et sur la cohérence interne, ne sont pas nécessairement définies.

En effet, on peut trouver dans ces systèmes des comportements socioéconomiques qui modifient l'organisation fonctionnelle et offrent diverses possibilités de relations entre les différents éléments et les niveaux d'organisations.

Ceci nous suggère deux réflexions, toutes deux liées à l'intensité du mécanisme sui-référentiel favorisant cette différenciation et cette territorialisation des systèmes locaux de la petite entreprise. La première réflexion nous amène à évaluer la persistance et la flexibilité de ce mécanisme sui-référentiel. La «décontextualisation» croissante de l'économie territoriale, du moins dans le cas italien n'est possible qu'à la condition d'annuler ou de vider de leur signification les phénomènes locaux de culture industrielle. Mais cette qualité sui-référentielle a été cependant suffisamment démontrée à partir de l'après-guerre; alors qu'on avait appuyé depuis longtemps sur la suprématie «idéologique» de la grande entreprise et sur le développement centralisé. La formation territoriale qui a caractérisé le développement économique italien a continué à croître même dans un contexte général de crise économique politique, nationale et internationale. Il n'est pas question de discuter ici quand les conditions de cette territorialité sont devenues fonctionnelles et sous quel type de développement; il n'est pas plus facile de dire aussi quand ce type de développement a correspondu à l'intérêt général. Mais on doit admettre, cependant, que ces changements ont servi de fonction stabilisatrice particulière, du point de vue économique et social, pour préserver les opportunités et atténuer les conflits. La recherche italienne qui a mis l'emphase sur ces systèmes locaux de petite entreprise ne peut s'expliquer par la situation théoriquement «excentrique» de ces systèmes, mais plus par l'évidence de leur performance.

Toutefois, il est clair que, même dans le cas pratésien, ces changements ne peuvent être considérés en marge de l'évolution de la division internationale du travail et demandent une nouvelle reconsidération des modèles socio-territoriaux. Cette nouvelle analyse d'un côté, se doit de confirmer la pertinence de tels modèles dans le processus général de transformation graduelle des «districts industriels marshalliens» d'origine vers des «systèmes socio-territoriaux». Cette évolution et, donc, la re-qualification de ces formations passe, en effet, par une ouverture cognitive sur l'extérieur qui implique un processus de réallocation productive et une certaine 
interaction avec la monoculture industrielle; ceci permet de comprendre cette caractéristique de contiguiité physique, présupposée par les économies d'agglomération typique du district industriel marshallien. Cette ouverture ou cette façon de faire permet de «libérer» de l'énergie réutilisable par les fonctions émergentes du tertiaire et des leaders.

Comme second élément de réflexion, on peut se poser la question politique sur la «désirabilité» ou du moins la sauvegarde de la qualité sui-référentielle de ces systèmes. L'auto-organisation, favorisant de nouvelles relations fonctionnelles de cohérence à l'intérieur des systèmes locaux de petite entreprise, peut finir par être objectivement gênée par la dynamique spatiale du tertiaire, en général, et du tertiaire supérieur, en particulier, qui favorise progressivement des phénomènes de concentration à l'encontre de la diffusion. Il semble que c'est une des menaces plus importante que la réutilisation de l'énergie libérée à l'intérieur du système; et l'expérience pratésienne du projet SPRINT montre l'insuffisance des ressources internes pour contrôler la situation. De telles ressources, si consistantes qu'elles soient, apparaissent encore largement potentielles et relèvent jusqu'à présent des caractéristiques reliées aux mécanismes auto-régulatifs auxquels on a déjà fait référence. Ces caractéristiques peuvent et doivent faire l'objet d'une évaluation politique. L'intégration étroite et typique de l'économique et du social (source réelle du mécanisme sui-référentiel) semble, en effet, garantir le maintien des différences spatiales dans l'optimisation de l'allocation des ressources; celles-ci semblent à la fois la condition pour générer de nouvelles relations dialectiques dans le processus de transformation qui ne soit pas dirigé de l'extérieur, et, à l'encontre de ce qui s'est passé dans le projet SPRINT, pour entraîner la participation. Cette capacité dialectique, bien que produite dans des conditions de grande incertitude et, par conséquent, exposée à bien des erreurs, semble pouvoir générer la complexité et la capacité pour maintenir et pour contrôler non seulement l'auto-conservation des «districts» mais aussi le fonctionnement plus général de chacune des variables socio-économiques cruciales nationales.

Il est naturel que ce jugement politique sur la désidérabilité de ces constructions industrielles historico-sociales doit être évalué selon la contingence de phénomènes plus importants, à l'échelle de réflexions plus larges et d'intérêts plus grands et par conséquent, plus «stratéqiques». Mais il est difficile de trouver une alternative au développement tertiaire localisé et relié à la monoculture industrielle. La qualité sui-référentielle demeure, en effet, ce patrimoine historique favorisant la cohésion sociale, les aptitudes communautaires au travail et la propension à l'entrepreneuriat, qui encadrent la formation de la richesse et qui constituent la seule sauvegarde pour une intégration fonctionnelle de le technologie afin d'économiser le travail et de favoriser l'échange systématique de l'information. 


\section{BIBLIOGRAPHIE}

Bagnasco, A, (1977), Tre Italie. Le problematiche territoriali dello sviluppo italiano, Bologne, II Mulino.

Balestri, A., Mutamento e politiche industriali nel distretto pratese, Milan, Angeli (à paraître).

Balestri, A.et Toccafondi, D., (1988), I produttori pratesi di tessuti, Prato, mimeo.

Becattini, G., (1979b), «Sopra alcune difficoltà dell' applicazione del concetto di mercato alla fenomenologia del lavoro», Bari, Actes du colloque sur «Il mercato del lavoro nel mezzogiomo d'Italia».

Becattini, G., (éd.), (1987), Mercato e forze locali : il distretto industriale, Bologne, Il Mulino.

Becattini, G., (éd.), (1989a), Modelli locali di sviluppo, Bologne, Il Mulino.

Becattini, G., (1989b), «Riflessioni sul distretto industriale marshalliano come concetto socioeconomico", Stato e Mercato, 25.

Bellandi, M., (1987), «La formulazione originaria», dans Becattini, op. cité.

Bellandi, M., (1989a), «Capacità innovativa diffusa e sistemi locali di impresa», dans Becattini (1989a).

Bellandi, M., (1989b), «Il distretto industriale e l'importanza di essere vicini», Florence, Faculté d'économie et de commerce, miméo.

Bertalanffy, L., (1968), General System Theory, New York, Braziller.

Brusco, S., (1989), «Quale politica industriale per il distretto industriale?», dans Becattini (1989a).

Dei Ottati, G., (1987), «ll mercato comunitario», dans Becattini (1987), op. cit.

Dematteis, G., (1989), «Contingenza ambientale e ordine economico : lo sviluppo locale in una prospettiva geograficas, dans Becattini (1989a), op. cit.

Garofoli, G., (1989), «Modelli locali di sviluppo : i sistemi di piccola impresa», dans Becattini (1989a), op. cit.

Giovannelli, L., (1982), «Distretto industriale e capacità inventiva : l'esempio di Prato», $L a$ confiuntura in Toscana, 1.

Giovannelli, L., (1984), Cambiamenti organizzativi e modelli organizzativi : il caso dell' industria tessile a Prato, Prato, Edizioni del Palazzo.

Hoover, E. M., (1948), The Location of Economic Activity, New York, Mc Graw Hill.

Lorenzoni, G., (1979), Una politica innovativa nelle piccole e medie imprese : l'analisi del cambiamento del sistema industriale pratese, Milan, Etas Libri.

Luhmann, N., (1983a), Illuminismo sociologico, Milan, Il Saggiatore, (ed. or. 1970).

Luhmann, N., (1983b), «Mutamento di paradigmi nella teoria dei sistemi», Sistemi urbani, 2.

Marshall, A., (1919), Industry and Trade, Londres, MacMillan.

Marshall, A., (1966), Principles of Economics, Londres, MacMillan, (ed. or. 1890).

Maturana, H. et Varela, F., (1980), Autopoiesis and Cognition. The Realisation of the Living, Dordrecht, Reidel.

Nigro, G., (1982), «Il caso Prato», dans G. Mori, (éd.), La Toscana, Turin, Einaudi.

Ortolani, M., (1963), Lombardia e Lancashire. Saggio di Geografia industriale comparata, Naple, CNR.

Pecora, A., (1960), La regione industriale del Lancashire. Problemi di geografia economica e umana, Naple, Atti Accademia Pontaniana, Vlll.

Pred, A. R.,(1978), Sviluppo industriale e sviluppo urbanonegliStati Uniti, Milan, Angeli(ed. or. 1966).

Prigogine I. et Nicolis, G., (1977), Self-Organisation in Nonequilibrium Systems, New York, Wiley and Sons. 
Pollard, A., (1982), Picking up the Threads : Small Firms in the Wool Textile Industry, Londres, ITGD.

Raffestin, C., (1984), «Territorializzazione, deterritorializzazione, riterritorializzazione e informazione», dans Turco, op. cité.

Rosemberg, M. (1982), Inside the Black Box. Technology and Economics, Cambridge, Cambridge University Press.

Russo, M., (1985), «Technical change and the industrial district : the role interfirm relations in the growth and transformation of the ceramic tile production in Italy", Research Policy, 16.

Sabel, C., (1982), Works and Politics. The Division of Labour in Industry, Cambridge, Cambridge University Press.

Storper, M. et Walker, R., (1983), «The theory of labour and the theory of location», International Journal of Urban and Regional Research, 7.

Tinacci Mossello, M., (1987a), «Economie di agglomerazione e sviluppo economico», dans Becattini (1987), op. cit.

Tinacci Mossello, M., (1987b), «Capacità innovativa nei distretti industriali. Formulazioni di ipotesi e verifica nel caso di Prato», Napoli, Convegno «Tecnologia e territorio», à paraître dans Del Monte et Viganovi, (éd.), Tecnologia e Territorio : esperienze regionali a confronto, Milan, Angeli.

Turco, A., (1984), (éd.), Regione e regionalizzazione, Milan, Angeli.

Turco, A., (1988), Verso una teoria geografica della complessità, Milan, Unicopli.

Vallega, A., (1976), Regione e Territorio, Milan, Mursia.

Vallega, A., (1982), Compendio di geografia regionale, Milan, Mursia.

Walker, R. et Storper, M., (1981), «Capital and industrial location», Progress in Human Geography, 5.

Zanfei, A., (1987), «Vincoli alla diffusione di tecnologie dell'informazione», Prato, Colloque sur «Prato 2000», miméo.

Zeleny, M., (1981), (éd.), Autopoiesis : The Theory of Living Organisation, New York, North Holland. 